\title{
« Ce n'est pas une crise des migrants mais une crise des politiques d'hospitalité »
}

\author{
Karen Akoka
}

26 juin 2017

Entretien - La notion de réfugié serait-elle neutre ? Non, pour la sociologue Karen Akoka, qui se penche sur son utilisation en France. La définition et les procédures d'octroi du statut n'ont cessé d'évoluer au gré de considérations politiques. Immanquablement, une politique migratoire restrictive se reflète sur le droit d'asile. Parler de " crise », ou du " problème de l'immigration ", en dit plus long sur nous que sur la réalité.

\section{Est-il juste de parler aujourd'hui de « crise des migrants ॥?}

Karen Akoka - Parler d'une crise des migrants en Europe voudrait dire qu'on serait face à un afflux inédit qui mettrait en danger les économies et les équilibres des pays du continent. Tous ceux qui travaillent sérieusement sur ces questions savent que l'Europe n'est pas dans un moment à ce point inédit et difficile (voir encadré). Surtout, les chiffres ne disent rien en soi. Pourquoi, ou par rapport à quoi, 1 million d'entrées serait beaucoup ? C'est notre usage des chiffres, la manière dont on les construit, dont on les sélectionne, dont on les mobilise, dont on les interprète qui est parlant. Et cela en dit long sur notre regard, sur notre représentation d'un fait social qui a existé de tout temps. C'est nous qui labellisons l'immigration comme un problème. Les uns pensent qu'on doit, malgré tout, prendre notre part du «fardeau », les autres qu'on ne peut pas l'assumer. Mais des deux côtés, on part d'un consensus largement questionnable : l'immigration pèse et pose problème. Les images diffusées par les médias viennent alimenter ces représentations en se focalisant sur le côté spectaculaire des arrivées - artificiellement concentrées dans les mêmes espaces cloisonnés qui fonctionnent comme des goulots d'étranglements - et en véhiculant l'idée que ce qui se passe à ces petites échelles est représentatif de l'échelle européenne tout entière.

Les propos alarmistes sur l'augmentation des demandes d'asile dans l'UE à 28 (de 500000 en 2014 à 1200000 en 2016) omettent de rappeler que des tendances similaires ont été atteintes au début des années 1990 suite aux guerres en ex-Yougoslavie avec 700000 demandes d'asile enregistrées en 1992 dans une UE alors à 15. Le nombre total de demandes déposées en 2016 ne représente quant à lui que $0,24 \%$ de ses 508 millions 
d'habitants. Et si on totalise toutes les demandes d'asile de ces dix dernières années, on reste malgré tout à $1 \%$. En France, l'augmentation de la demande observée ces trois dernières années (+ 20000 demandeurs d'asile en 2015 par rapport à 2011) est dérisoire. Sans compter que le nombre de 87000 enregistré en France en 2016 et présenté comme un pic historique, a en fait déjà été atteint : il descend à 63000 si on enlève les mineurs accompagnants soit les mêmes chiffres qu'en 1989 (où ces mineurs n'étaient pas comptés).

On oublie aussi de rappeler que les chiffres de l'immigration régulière sont stables en France depuis près de trois décennies.

On oublie aussi de rappeler que les chiffres de l'immigration régulière sont stables en France depuis près de trois décennies (autour de 200000 par an). Ou encore que l'augmentation du nombre des entrées clandestines en Europe (multipliées par quatre entre 2014 et 2017) reflète aussi d'autres réalités : les restrictions des voies d'immigration légales et l'augmentation des moyens de l'agence qui interpelle puis compte les migrants (Frontex), dont le budget a été multiplié par trois sur la même période (de 100 à 300 millions d'euros). Sans parler du chiffre d'un million de migrants clandestins entrés en Europe en 2016 (parmi lesquels on retrouve les demandeurs d'asile précités), qui est largement surévalué. Frontex compte en effet le nombre de franchissements et non le nombre de personnes ayant franchi les frontières extérieures de l'UE. Les mêmes individus sont souvent comptabilisés plusieurs fois : après être entrés par la Grèce, leur parcours les amène souvent à en sortir par les Balkans, pour y entrer à nouveau via la Croatie ou la Hongrie. En outre, on parle toujours des flux, jamais des soldes migratoires (entrées moins sorties), qui donnent une image plus juste de l'immigration, les sorties étant loin d'être négligeables. Rappelons enfin que le monde comptait davantage de migrants au début du $X X^{\mathrm{e}}$ siècle qu'aujourd'hui et la France plus d'étrangers dans l'entre-deuX-guerres que de nos jours.

De même, il est faux de parler d'un Il est faux de parler d'un déséquilibre économique causé par l'immigration. déséquilibre économique causé par l'immigration. L'Europe vieillissante a besoin d'équilibrer sa démographie notamment pour payer les retraites et les dépenses de santé des plus âgés, postes très lourds dans nos budgets nationaux[1]. Nombre de chercheurs ont montré que l'immigration était très loin de représenter le fardeau dépeint dans les médias et qu'elle aurait même des incidences positives sur les Pib dans les pays occidentaux : ce que les migrants rapporteraient en effet (via les impôts, tva, charges, consommation) serait supérieur à ce qu'ils coûteraient (chômage, maladie, etc.). Ils ont aussi écarté tout lien de causalité mécanique entre chômage et immigration. Cette image, des plus répandues, repose sur l'idée erronée de notre marché du travail comme un gâteau fixe à se partager : plus on serait nombreux, plus il faudrait se serrer la ceinture. Or le gâteau, surtout dans une économie de services, s'agrandit avec l'arrivée de nouvelles personnes qui consomment, produisent et créent des services, embauchent même, et sont donc aussi sources de demandes[2]. 
Si le moment est inédit, c'est davantage en raison des dispositifs de contrôle, de fermeture des frontières.

Quand les historiens se pencheront sur la période actuelle, ils se demanderont quelle était notre rationalité : pourquoi avoir dépensé autant d'argent, conduit à la mort des dizaines de milliers d'individus, gaspillé des vies laissées en errance à cause de la fermeture des frontières alors que le principe de solidarité aurait dû nous conduire au choix de l'entraide et que d'un point de vue pragmatique, nous avons « besoin » de ces populations, comme nous aurons besoin de solidarité, le jour où le vent tournera ? II n'y a pas de crise des migrants, ni de crise des réfugiés, mais bien une crise des politiques d'hospitalité et de solidarité. Si le moment est inédit, c'est davantage en raison des dispositifs de contrôle, de fermeture des frontières qui n'ont fait que s'accentuer ces dernières décennies jusqu'au pic actuel. Les passeports ont été inventés après la première guerre mondiale. Avant cette date, l'Europe occidentale formait un espace ouvert aux déplacements transfrontaliers. La libre circulation n'est pas l'utopie insensée qu'on raconte : elle a d'une certaine manière déjà existé et n'a pris fin qu'il y a cent ans. Elle existe d'ailleurs encore aujourd'hui, mais seulement pour une partie de la planète.

\section{Comment se sont forgées les politiques d'asile?}

Pour parler de l'histoire de l'asile, on construit généralement un récit linéaire. Mais en racontant l'histoire de ceux que l'on a désignés comme réfugiés, on laisse de côté tous les autres. Tenir ensemble les inclus et les exclus de cette catégorie permet de comprendre comment, à chaque période se décide qui est réfugié et qui ne l'est pas, et donc de sortir du récit linéaire pour mieux voir ce qui est de l'ordre de l'arbitraire ou relève des considérations politiques, économiques, juridiques de la société d'accueil dans la désignation comme réfugié.

Il faut ainsi attendre que la religion protestante devienne légitime sur le sol européen (à la fin du XVIIII siècle) pour que les huguenots qui avaient fuient la France de la révocation de l'édit de Nantes (1685) entrent dans les dictionnaires anglais et français comme réfugiés. Mais les juifs et les maures d'Espagne qui avaient fui un siècle auparavant pour les mêmes raisons (religieuses) parfois vers les mêmes pays (Hollande), n'entreront jamais dans le langage courant comme « réfugiés ».

Dans l'entre-deux-guerres, les définitions du réfugié s'appliquent uniquement à des groupes nationaux ou ethniques, pas à des individus. C'est la SDN qui décide quels groupes sont concernés. Le groupe le plus emblématique étant celui des Russes. Même si la plupart de ceux qui partent fuient la très grande famine qui sévit à l'époque, tout Russe qui a quitté la Russie est considéré comme réfugié sur la base des politiques de dénationalisation pratiquées par le nouveau pouvoir bolchévique à l'encontre de ses exilés. Au même moment, ni les juifs de Hongrie dénationalisés par le nouvel État-Nation, ni les Italiens puis les Espagnols fuyant les régimes fascistes de Mussolini et Franco (et pour certains dénationalisés), ne peuvent bénéficier du statut de réfugié : leur groupe n'a pas été désigné comme tel par la SDN. Ces choix sont largement politiques. Dans les années 1920, les velléités expansionnistes de la révolution bolchévique sont davantage un problème public que le fascisme. Et lorsqu'il le devient, dans les années 1930, c'est essentiellement en référence à l'Allemagne nazie, tandis que Salazar, Mussolini et Franco 
restent des interlocuteurs des démocraties occidentales. Les juifs allemands devront attendre la fin des tentatives de conciliation avec Hitler et le retrait allemand de la SDN, en 1938, pour pouvoir être considérés comme un groupe de réfugiés.

Après guerre, en 1951, la convention de Genève institue le réfugié comme quelqu'un qui craint des persécutions. On y voit aujourd'hui une définition claire et neutre. Pourtant elle aurait pu être très différente : la sociologue américaine Jacqueline Bhabha indique que le bloc occidental voulait faire du réfugié la victime de persécutions et de violences politiques face aux soviétiques qui défendaient l'idée d'un réfugié comme victime de violences sociales et économiques. La première conception, issue des Lumières, juge plus grave de toucher aux droits politiques, civiques et individuels qu'aux droits économiques et sociaux collectifs. C'est cette hiérarchie des valeurs qui a prévalu dans la convention de Genève en 1951. Si la conception soviétique avait été retenue, ceux que l'on appelle « migrants économiques » seraient peut-être considérés comme des « réfugiés de la faim » et les réfugiés légitimes d'aujourd'hui comme des « migrants politiques » tentant de se faire passer pour des réfugiés...

\section{La convention de Genève définit le réfugié comme une personne qui « craint avec} raison d'être persécutée du fait de sa race, de sa religion, de sa nationalité, de son appartenance à un certain groupe social ou de ses opinions politiques ॥. De tels critères permettent-ils d'assurer une continuité et une cohérence dans la figure du réfugié depuis 1951 ?

Karen Akoka - Le droit laisse toujours place à interprétation et la convention de Genève ne fait pas exception. Jusqu'au milieu des années 1980, lorsqu'une grande partie des étrangers qui demandaient l'asile venaient de pays sous domination soviétique (ex-URSS et démocraties populaires) ou communistes (Vietnam, Laos, Cambodge), le statut de réfugié était accordé quasi automatiquement sans qu'on examine vraiment les raisons des départs. Dans la Convention, il est écrit qu'il faut craindre « avec raison » d'être persécuté mais non pas qu'il faut craindre d'être « individuellement » persécuté. La recherche de persécution individuelle restait alors marginale : elle était appliquée à certains groupes que l'on cherchait à exclure du statut, généralement pour ménager les relations diplomatiques avec leurs pays d'origine.

Les années 1980 sont des années de transition : on peut alors parler d'un asile à deux vitesses, avec la désignation par groupe pour certaines nationalités (désirables) et l'exigence de la preuve d'une persécution individuelle pour d'autres. Finalement, dans le courant des années 1990, l'impératif de la personnalisation de la crainte de persécution se généralise. Si au début il suffit d'être kurde pour obtenir le statut, dès le milieu des années 1990, être kurde ne suffit plus : il faut montrer qu'on est individuellement persécuté comme kurde et que l'on n'a pas eu recours à la lutte armée ou la violence pour défendre sa cause (ce qui peut, dans certains cas, constituer une injonction contradictoire). D'où une chute du taux d'accord pour les demandeurs d'asile turcs dans le contexte du renforcement des alliances de la Turquie avec les pays occidentaux (qui s'exprime notamment par des perspectives nouvelles d'intégration dans l'Europe) grâce au rôle stratégique joué par Ankara pendant la première guerre du Golfe. 
Au-delà de cet exemple, on peut voir plusieurs causes à cette généralisation de l'individualisation et à l'accroissement des exigences, qui mènent mécaniquement à la montée des rejets. Avec la fin de la guerre froide et de la bipolarité, il n'est plus utile de désigner comme réfugiés des groupes fuyant des pays communistes. Les années 1990 voient aussi le renforcement de l'idée de l'immigration comme problème, et des politiques d'immigration restrictives apparaissent, qui bouleversent les politiques d'asile. Enfin, à la même période, le nombre de demandeurs issus d'anciens pays colonisés - avec lesquels les ex-puissances coloniales tentent de mettre en place ou de consolider de bonnes relations économiques, sur le modèle de la France-Afrique - a augmenté. Donner le statut est devenu non seulement inutile, mais contreproductif.

$\mathrm{Si}$

La grande majorité des exilés fuient une situation imbriquée, incluant l'individuel et le collectif.

l'Union européenne a récemment réintroduit une dimension plus collective pour les civils qui fuient les conflits armés, en créant la " protection subsidiaire » comme alternative au statut de réfugié, c'est bien parce que la convention de Genève est systématiquement interprétée du point de vue individuel. Et cette protection subsidiaire ne donne pas le statut de réfugié : elle n'a qu'une durée limitée. La grande majorité des exilés fuient une situation imbriquée, incluant l'individuel et le collectif, ce que les institutions qui statuent sur leur demande ne reconnaissent pas.

\section{En quoi les politiques d'asile et d'immigration sont-elles liées ?}

Karen Akoka - Les étrangers se sont longtemps dirigés vers la catégorie de réfugié ou de migrant en fonction de leurs besoins et d'une série de critères à la fois collectifs et subjectifs. Et les pouvoirs publics, eux aussi, ont souvent poussé tel ou tel groupe vers les procédures d'asile ou d'immigration en fonction de présupposés et de considérations politiques, économiques et surtout diplomatiques.

Dans les années 1960, le représentant du ministère des Affaires étrangères au conseil d'administration de l'Ofpra [Office français de protection des rapatriés et apatrides] a ainsi signalé à plusieurs reprises : "II y a trop de réfugiés yougoslaves, ce n'est pas bon pour nos relations diplomatiques. II faut en rejeter davantage ». Et le nombre d'admis au statut a rapidement chuté. Deux ou trois ans plus tard, des accords de main-d'œuvre étaient négociés avec la Yougoslavie. Ayant davantage accès aux procédures d'immigration, les Yougoslaves n'ont plus guère demandé l'asile. Ce qui a satisfait tout le monde. C'est à peu près la même histoire pour les Espagnols et les Portugais. Durant les années 1960 et 1970, ceux d'entre eux qui demandaient le statut de réfugié connaissaient un régime un peu plus dur que leurs homologues venus de pays communistes. Mais ces deux nationalités étaient aussi les premières à recevoir des permis de séjour au titre de l'immigration, car elles étaient considérées « plus assimilables » que les maghrébins ou subsahariens. On voulait bien d'eux comme migrants mais pas comme réfugiés pour ne pas nuire aux relations diplomatiques avec leur pays d'origine.

À l'inverse, dans les années 1980, le gouvernement a fait venir lesboat people de la péninsule indochinoise selon des critères qui n'avaient rien à voir avec la convention de Genève (services rendus ou liens avec la France) : on leur a ouvert des aides inédites à 
condition qu'ils optent pour le statut de réfugié, tout en l'accordant à tous ceux d'entre eux qui le demandaient (les taux étaient alors de 99,98 \%). On voulait d'eux comme réfugiés et pas comme migrants. Les accueillir comme réfugiés permettait de dénoncer les régimes communistes de la péninsule tout en procurant une main d'œuvre jugée docile à certaines industries qui en avaient besoin. Les accueillir comme migrants aurait risqué de remettre en question la décision déjà prise de fermer l'immigration de travail.

« Être réfugié ou migrant se construisait au croisement des dispositifs d'action publique et des décisions des étrangers, selon des critères qui débordaient la question de la persécution.»

Les exilés, eux aussi, pouvaient davantage choisir de se tourner vers l'un ou l'autre dispositif en fonction de leurs besoins et contraintes propres. Certains s'abstenaient de demander le statut de réfugié, vécu comme une rupture symbolique trop forte avec le pays d'origine, ou parce qu'il interdit les retours pour de courts séjours, à l'occasion d'un décès ou d'un mariage par exemple. D'autres, au contraire, faisaient ce choix parce qu'ils y voyaient une manière de dire leur résistance au régime de leur pays, ou de se projeter vers un retour plus que vers l'installation dans le pays hôte. Être réfugié ou migrant se construisait ainsi au croisement des dispositifs d'action publique et des décisions subjectives des étrangers, selon des critères qui débordaient largement la question de la persécution. C'est à cette porosité qui laissait une certaine latitude - aux exilés pour s'autodéfinir et aux pouvoirs publics pour gérer les complications qu'un statut éminemment politique ne pouvait manquer de créer dans les relations diplomatiques - qu'a mis fin le renforcement des politiques migratoires restrictives. La porosité des dispositifs d'asile et d'immigration reflétait la porosité des catégories et des identités. La rigidification des procédures d'immigration a forcément entraîné celle des procédures d'asile. Au final les dispositifs clôturés, nés de trois décennies de politiques d'asile et d'immigration restrictives, comme la surenchère des exigences ont obligé à des assignations rigides qui ne correspondent en rien aux trajectoires et à l'expérience intime des exilés ; tout en légitimant leur exclusion. II n'y a pas de politique d'asile qui tienne sans une politique d'immigration ouverte en amont pour laisser les gens atteindre le territoire et en aval pour garantir un système souple. On ne peut pas se battre pour les réfugiés si on ne se bat pas pour les migrants : les catégories sont poreuses et ont besoin de dispositifs poreux pour les refléter. Cet argument fait bien sûr débat au sein du milieu associatif. Certains pensent qu'il faut sauver ceux qu'on peut encore sauver, c'est-à-dire ceux qui sont encore légitimes, en les maintenant bien à distance de ceux qui ne le sont pas, quitte à entériner leur illégitimité. Pourtant, jouer les uns contre les autres est un piège. Aujourd'hui l'asile sert de caution aux politiques migratoires restrictives, tout en étant lui-même de plus en plus restreint. À la fin, tout le monde y perd. II y a un impératif à la fois intellectuel et politique à ne pas croire et faire croire en ces dichotomies essentialistes et en cette hiérarchie des légitimités.

Aujourd'hui l'asile sert de caution aux politiques migratoires restrictives, tout en étant lui-même de plus en plus restreint.

En sociologie, on distingue la catégorie sociale, nommée de l'extérieur, du groupe social, entité qui se nomme elle-même. Les travaux de Luc Boltanski ont par exemple montrés comment les cadres se sont constitués en groupe social. D'une certaine manière, les 
réfugiés étaient un groupe social pendant plusieurs décennies, et sont devenus une catégorie sociale à partir des années 1980. La convention de Genève a été en partie écrite par des réfugiés, notamment les réfugiés russes qui y avaient intérêt. En France, dans les années 1950-1960, voire 1970, les agents de l'Ofpra étaient des réfugiés qui donnaient le statut à leurs compatriotes dans la même situation qu'eux. Le chef de la section russe était un ancien diplomate russe d'avant la révolution de 1917, le chef de la section arménienne un réfugié arménien, etc. Mais le profil des agents de l'Ofpra s'est progressivement transformé (français, pour partie fonctionnaires), à mesure que l'on allait vers une conception plus rigide du réfugié, devant répondre à des critères définis de l'extérieur.

La nature fluctuante des définitions et des interprétations souligne bien qu'au final, la qualité de réfugié réside moins dans le vécu ou l'histoire de la personne que dans le processus de désignation.

Pour

moi, un Pour moi, un réfugié n'est, ni plus ni moins, que quelqu'un qui a réussi à obtenir le statut. réfugié

n'est, ni plus ni moins, que quelqu'un qui a réussi à obtenir le statut. Cette définition reflète une réalité institutionnelle et permet d'éviter d'entrer dans la question inextricable du vrai ou du faux, ou autrement dit permet d'éviter la préemption essentialiste selon laquelle il y a une qualité intrinsèque de réfugié, des réfugiés en soi, quasi naturels qui pourraient se reconnaître

Pour désigner ceux qui ne sont pas reconnus réfugiés (parce qu'ils ne le demandent pas ou parce qu'ils échouent à l'être), je défends l'utilisation du terme " exilés » : peu importe la raison pour laquelle les personnes sont parties, on reconnaît le départ et l'arrachement qu'il induit.

\section{Les officiers de l'Ofpra, comme les juristes des associations, ne croient-ils pas appliquer une définition neutre?}

Karen Akoka - J'ai travaillé au HCR, et pendant des années j'ai cru que cette définition était neutre. J'ai aussi essentialisé les réfugiés, cru qu'il y avait une différence objective et presque ontologique entre réfugiés et migrants et qu'il ne fallait surtout pas les mélanger. Présenter la catégorie de réfugié comme juridique et neutre est un leurre : elle est éminemment politique. Comme les Indochinois d'hier, les Syriens sont aujourd'hui considérés comme les réfugiés les plus légitimes. Le réfugié syrien est triplement légitime dans nos représentations actuelles : il appartient aux classes moyennes qui nous ressemblent, il fuit à la fois Bachar El Assad - le dictateur par excellence - et Daech l'ennemi global par excellence. Au-delà du cas syrien, il semblerait que le fondamentalisme islamique ait, d'une certaine manière, remplacé le communisme comme moteur dans le processus de désignation du réfugié. Alors que l'accès au statut de réfugié est aujourd'hui de plus en plus restreint, de nouvelles catégories - qui en étaient exclues - se voient pourtant ouvrir le bénéficie du statut : les victimes de violences liées à leur genre ou à leurs orientations sexuelles (excision, mariage forcé, persécution des homosexuels), souvent identifiées (et à tort) comme des violences relevant du fondamentalisme islamique. Pour autant, l'ensemble de l'immigration étant perçu comme problématique, le fait d'être « favorisé » pour l'accès au statut reste aujourd'hui relatif. Dans la mesure où l'immigration 
est désormais construite comme un problème, on ne traite pas les Syriens (où les victimes de violences liées à leur genre) de la même manière que les autres groupes légitimes d'hier. Alors que l'on a fait venir les Indochinois par avion (plus de 1000 personnes par mois pendant 5 ans), les Syriens doivent atteindre l'UE contre vents et marées pour pouvoir demander l'asile. On cherche à les en empêcher par tous les moyens. C'est seulement une fois qu'ils sont là, qu'ils sont assez facilement reconnus comme réfugiés.

L'ensemble de l'immigration étant perçu comme problématique, le fait d'être « favorisé » pour l'accès au statut reste aujourd'hui relatif.

Le principe pour les institutions - le HCR, l'Ofpra ou ses homologues dans d'autres pays -, c'est la recherche de la persécution individuelle. Une recherche qui n'est pas anodine parce qu'elle est le plus souvent au service du rejet des demandes d'asile. Ces agents étatiques ou internationaux cherchent l'étranger individuellement persécuté, avec en tête, plus ou moins consciemment, la figure du dissident politique des années de guerre froide, qui représente d'une certaine façon l'archétype du réfugié ou le réfugié « idéal ». Or les dissidents politiques et les personnes individuellement visées étaient bien moins nombreux que celles qui avaient fui un conflit généralisé, un régime qu'elles exécraient, ou un système, politique ou économique, dans lequel elles ne voulaient tout simplement pas vivre. L'image du réfugié - militant politique et personnellement recherché - revisitée, glorifiée, mais largement éloignée de la réalité de ce que fut la demande d'asile des années cinquante à quatre-vingt, a peu à peu dessiné en creux celle du faux demandeur d'aujourd'hui. On le suspecte d'être un « faux réfugié », alors que ce sont nos exigences qui ont augmenté. Cette recherche par les officiers de l'Ofpra, de réfugiés quasi introuvables parmi les demandeurs d'asile, conduit à un taux très important de rejets et à un cercle vicieux dans lequel la surenchère des exigences entraîne une surenchère des fraudes, justifiant une nouvelle surenchère d'exigences et de contrôles.

\section{Que pensez-vous du terme « réfugiés climatiques ॥?}

Karen Akoka - II est intéressant de voir que l'on utilise plus facilement le terme « réfugié climatique » que " réfugié de la faim » alors que ces deux critères sont tout autant absents de la convention de Genève. Cela en dit plus long sur « nous » que sur " eux », ceux qu'on appelle les « réfugiés ». Car cette appellation traduit la légitimité politique que la question climatique est en train d'acquérir dans nos sociétés. C'est sans doute parce que l'on commence à reconnaître notre part de responsabilité dans le changement climatique que l'on a plus de facilité à parler de réfugiés climatiques. S'agissant d'exilés qui fuient la faim ou l'absence de perspectives socioéconomique dans leur pays, nous sommes bien moins prêts à reconnaître notre responsabilité. Les inégalités Sud-Nord ou les situations de nombre de pays en développement ne sont pourtant pas sans liens avec les héritages coloniaux, postcoloniaux ou l'impact des politiques d'ajustement structurel imposées par la gouvernance internationale depuis les années 1980. Mais on les impute uniquement à la mauvaise gestion de ces pays en interne. Nos dénominations sont bien le reflet de constructions politiques. Est-ce un hasard si l'on parle de violences politiques mais pas de violences économiques (on utilise davantage l'expression « inégalités économiques »), de « faim » plutôt que de " d'insécurité alimentaire », dès lors que l'exil politique (ou climatique) est plus légitime que l'exil économique? 
Des propos recueillis par Marine Carlier et Solange de Coussemaker

[1] Cf. par ex. Elena Ambrosetti et Cristina Giudici, "L'Europe rajeunie par ses migrants ", Revue Projet 335 [NDLR]

[2] Cf. par ex. Damien de Blic, "Les immigrés, fardeau ou manne économique ? ", Revue Projet, 335 [NDLR] 